\title{
Homomorphisms and Direct Sums of Nested Hilbert Spaces
}

\author{
A. Grossmann * \\ Institut des Hautes Etudes Scientifiques \\ 91. Bures-sur-Yvette - France \\ Received June 10, 1966
}

\begin{abstract}
We prove some elementary facts about homomorphisms and infinite direct sums of nested Hilbert spaces.
\end{abstract}

\section{Introduction}

In a previous paper [1] we have studied a class of vector spaces endowed with a certain "self-dual" structure. The applications to follow - as well as mathematical decorum - require the introduction of suitable definitions concerning isomorphisms, subspaces, group representations etc.

All these concepts can be obtained in a standard way [2] from that of homomorphism; so our first task is to single out homomorphisms among all operators between nested Hilbert spaces.

For the special case of chains of Hilbert spaces, the natural definition can be found in PALAIS ${ }^{1}$ [3]. The generalization to arbitrary nested Hilbert spaces is studied in Sections 2a to $2 \mathrm{c}$ below. It is preceded by a discussion of relevant results from [1] (Sections 1 a to $1 \mathrm{e}$ ).

The last part of the paper (Sections $3 a$ to $3 b$ ) deals with infinite direct sums of nested Hilbert spaces. The results are used in the accompanying paper which is concerned with quantized fields.

\section{Preliminaries}

The definition of nested Hilbert space is recalled in Section $1 \mathrm{a}$; that of operator and of adjoint operator in Section $1 \mathrm{~b}$. The reader should keep in mind the definition of the set $J(A)$ which describes the "regularity" of the operator $A$ with respect to the space $H_{I}$. The larger $J(A)$, the "better" or "smoother" $A$.

Operators between nested Hilbert spaces behave as bounded operators between Hilbert spaces as far as addition, multiplication by scalars and the taking of adjoints go. The product of $n$ operators is defined if and only if the sets $J\left(A^{(j)}\right)(j=1, ., n)$ satisfy a certain condition (Section 1c).

* Present Address: Physique Theorique, Université Aix-Marseille.

1 I am indebted to D. RUELLE who pointed out this reference. 
All results about products and matrix elements are special cases of results about operators (Section $1 \mathrm{~d}$ ). This is so because of the existence of a canonical bijection between vectors $h \in H_{I}$ and linear operators $|h\rangle$ from complex numbers into $H_{I}$. The (operator) adjoint of $|h\rangle$ is denoted by $\langle h|$. Since the correspondence $|h\rangle \leftrightarrow\langle h|$ is bijective we are perhaps closer to the Dirac bracket formalism than is RoBERTs [4].

\section{a) Nested Hilbert space}

A nested Hilbert space $H_{I}$ is an algebraic inductive limit of a family $H_{r}(r \in I)$ of Hilbert spaces, taken with respect to a family $E_{s r}(r \in I$, $s \geqq r$ ) of linear bounded injective operators with dense range (nestings). The directed set $I$ is endowed with an order-reversing involution $r \leftrightarrow \bar{r}$. There exists a $o \in I$ such that $\bar{o}=0$. The natural embedding of $H_{r}$ into $H_{I}$ is denoted by $E_{I r}$.

If $s \geqq r$, then $E_{I s} H_{s} \supseteqq E_{I r} H_{r}$. It is assumed:

$\left(N H_{1}\right)$. If $r, q$ are any two elements of $I$, then there exists a $p \leqq r, q$ such that $E_{I p} H_{p}$ is the intersection of $E_{I r} H_{r}$ and of $E_{I q} H_{q}$.

$\left(\mathrm{NH}_{2}\right)$. There exists a family $u_{\bar{r} r}$ of unitary mappings from $H_{r}$ onto $H_{\bar{r}}$ such that $u_{\mathrm{oo}}=1$ and that, for every $r \in I$ and for every $s \geqq r$, one has

$$
\left(E_{s r}\right)_{r s}^{*}=u_{r \bar{r}} E_{\bar{r} \bar{s}} u_{\bar{s} s} \text {. }
$$

Here $\left(E_{s r}\right)_{r s}^{*}$ is the Hilbert space adjoint of $E_{s r}$.

\section{b) Operators}

Let $H_{I}$ and $F_{I^{\prime}}$ be two nested Hilbert spaces. For every $r \in I$ and every $r^{\prime} \in I^{\prime}$ consider the Banach space $L\left(r ; r^{\prime}\right)$ of all bounded linear operators from the Hilbert space $H_{r}$ into the Hilbert space $F_{r^{\prime}}$. If $r \geqq s$ and $r^{\prime} \leqq s^{\prime}$, then one has the natural maps

$$
A_{r^{\prime} r} \rightarrow E_{s^{\prime} r^{\prime}} A_{r^{\prime} r} E_{r s}
$$

of $L\left(r ; r^{\prime}\right)$ into $L\left(s ; s^{\prime}\right)$. An operator from $H_{I}$ into $F_{I^{\prime}}$ is defined as an element of the algebraic inductive limit $L\left(H_{I} ; F_{I^{\prime}}\right)$ of the spaces $L\left(r, r^{\prime}\right)$ with respect to the maps (1.1).

To every $A \in L\left(H_{I} ; F_{I^{\prime}}\right)$ one associates the set $J(A)$ of all pairs $\left\{r, r^{\prime}\right\}$ such that $A$ has a representative $A_{r^{\prime} r}$ in $L\left(r ; r^{\prime}\right)$ (i.e. such that $A$ defines a bounded map from $H_{r}$ into $F_{r^{\prime}}$ ).

An operator $A \in L\left(H_{I} ; F_{I^{\prime}}\right)$ need not be defined on all ${ }^{2}$ of $H_{I}$. Its domain is the union

$$
\bigcup_{r \in D(A)} E_{I r} H_{r}
$$

2 The notation $L\left(H_{I} ; F_{I^{\prime}}\right)$ may be misleading since $L(X ; Y)$ is commonly used to denote the set of continuous linear maps from all of $X$ into $Y$. 
where he initial subset $D(A) \leqq I$ is defined as

$$
D(A)=p r_{I} J(A)
$$

i.e. as the projection of $J(A)$ on $I$. One introduces also a final subset $R(A) \leqq I^{\prime}$ defined by

$$
R(A)=p r_{I^{\prime}} J(A)
$$

i.e. as the projection of $J(A)$ on $I^{\prime}$.

It is important to realize that our definitions leave no freedom in the choice of the domain of $A$. The reason is, roughly speaking, that a family of admissible domains is built into the structure of $H_{\mathrm{I}}$; such domains are unions of subspaces of the form $E_{\mathrm{I} r} H_{r}$. To define $A$ as an element of the inductive limit $L\left(H_{\mathrm{I}} ; F_{\mathrm{I}^{\prime}}\right)$ means essentially to choose the maximal admissible domain, i.e. the union of all subspaces $E_{\mathrm{Ir}} H_{r}$ each of which is mapped by $A$ into some $E_{\mathrm{I}^{\prime} r^{\prime}} F_{r^{\prime}}$.

An operator is fully determined by any one of its representatives, which are bounded operators between Hilbert spaces. If $\mathrm{A}_{r^{\prime} r}$ is the $\left\{r, r^{\prime}\right\}$-representative of $A$, we shall write $A=E_{2}\left(I \times I^{\prime} ; r, r^{\prime}\right) A_{r^{\prime} r}$.

Every $A \in L\left(H_{\mathrm{I}} ; F_{\mathrm{I}^{\prime}}\right)$ has a unique adjoint $A^{*} \in L\left(F_{\mathrm{I}^{\prime}} ; H_{\mathrm{I}}\right)$. The set $J\left(A^{*}\right)$ consists precisely of the pairs $\left\{r^{\prime}, r\right\}$ such that $\left\{\bar{r}, \bar{r}^{\prime}\right\} \in J(A)$. The representative $\left(A^{*}\right)_{r} r^{\prime}$ is given by

$$
\left(A^{*}\right)_{r r^{\prime}}=u_{r \bar{r}}\left(A_{\bar{r}^{\prime} \bar{r}^{\prime}}\right)_{\bar{r} \bar{r}^{\prime}} u_{\bar{r}^{\prime} r^{\prime}} .
$$

One has $A^{* *}=A$ for every $A \in L\left(H_{\mathrm{I}} ; F_{\mathrm{I}^{\prime}}\right)$. If, in particular, $A$ is an operator in a nested Hilbert space, i.e. if $A \in L\left(H_{\mathrm{I}} ; H_{\mathrm{I}}\right)$, then $A^{*}$ also belongs to $L\left(H_{\mathrm{I}} ; H_{\mathrm{I}}\right)$.

\section{c) Products}

Let $A^{(1)} \in L\left(H_{\mathrm{I}}^{(1)} ; H_{\mathrm{I}}^{(2)}\right), \quad A^{(2)} \in L\left(H_{\mathrm{I}}^{(2)} ; H_{\mathrm{I}}^{(3)}\right), \ldots, A^{(n)} \in L\left(H_{\mathrm{I}}^{(n)} ;\right.$ $\left.H_{\mathrm{I}}^{(n+1)}\right)$. The product $A^{(n)} \ldots A^{(1)}$ is defined if and only if there exists a "chain" of $n+1$ elements $r^{(1)} \in I^{(1)}, r^{(2)} \in I^{(2)}, \ldots, r^{(n+1)} \in I^{(n+1)}$ such that

$$
\left\{r^{(1)}, r^{(2)}\right\} \in J\left(A^{(1)}\right),\left\{r^{(2)}, r^{(3)}\right\} \in J\left(A^{(2)}\right), \ldots,\left\{r^{(n)}, r^{(n+1)}\right\} \in J\left(A^{(n+1)}\right) .
$$

It belongs to $L\left(H_{\mathrm{I}}^{(1)} ; H_{\mathrm{I}}^{(n+1)}\right)$. Its $\left\{r^{(1)}, r^{(n+1)}\right\}$-representative is $A_{r^{(n+1)} r^{(n)}}^{(n)} A_{r^{(n)} r^{(n-1)}}^{(n-1)} \ldots A_{r^{(2)}}^{(1)} r^{(1)}$.

This definition requires a remark about associativity.

In order to simplify notation, consider the case $n=3$. If the product $C B A$ is defined, then $C B$ and $B A$ are defined, and so are the products $C(B A)$ and $(C B) A$ which are of course equal to $C B A$. It can happen, however, that the products $B A$ and $C(B A)$ (two factors each) are defined, while the product $C B A$ of three factors is not defined. Examples can be found in [1]. 
In other words: Parentheses may be inserted but may not always be removed.

\section{d) Scalar product and matrix elements}

Consider, in particular, the case that one of the spaces is the set $C$ of complex numbers considered as nested Hilbert space. For every $h \in H_{I}$, denote by $|h\rangle$ (or by $|h ; C\rangle$ if there is danger of confusion) the map $\varphi \rightarrow \varphi h(\varphi \in C)$, considered as an element of $L\left(C ; H_{I}\right)$. Every element of $L\left(C ; H_{I}\right)$ can be written in this way. Write $\langle h|$ to denote the adjoint of $|h\rangle$.

The product $\langle g \mid h\rangle\left(h \in H_{I}, g \in H_{I}\right)$ of operators (which is defined if and only if $\bar{J}(f) \cap J(g)$ is not empty) is a linear mapping of $C$ into itself. One can identify this mapping with the value it takes at $\varphi=1$ and call the number $\langle g \mid h\rangle$ the scalar product of the vectors $g$ and $h$. If $o \in J(g)$ and o $\in J(h)$, then $\langle g \mid h\rangle$ is the scalar product in $H_{0}$.

If $h \in H_{I}$ and $f \in F_{I^{\prime}}$ are arbitrary, then the product $|h\rangle\langle f|$ is defined. It belongs to $L\left(F_{I^{\prime}} ; H_{I}\right)$.

If $A \in L\left(H_{I} ; F_{I^{\prime}}\right), h \in H_{I}$ and $f \in F_{I^{\prime}}$, then $\langle f|A| h\rangle$ denotes the product of the three operators $\langle f|, A$ and $|h\rangle$. It is defined if and only if the intersection of $\bar{J}(f) \times J(h)$ and of $J(A)$ is not empty. It is, again, a linear mapping of $C$ into itself; if one identifies it with the value taken at $\varphi=1$ it is called the matrix element of $A$ between $f$ and $h$. If $\left\{\mathrm{o}, \mathrm{o}^{\prime}\right\} \in J(A)$, $\mathrm{o} \in J(h)$, o' $\in J(f)$ then $\langle f|A| h\rangle=\left(f_{0^{\prime}}, A_{0^{\prime}{ }_{0}} h_{0}\right)$ is the Hilbert space matrix element of $A_{\mathrm{o}^{\prime} \mathrm{o}}$.

While $\langle f|A| h\rangle$ is a product of three factors, $\langle f \mid A h\rangle$ is a product of two factors. So it can happen that $\langle f \mid A h\rangle$ is defined and $\langle f|A| h\rangle$ is not.

e) Correspondence with operators in $H_{0}$

Let $A \in L\left(H_{I} ; H_{I}\right)$. If $J(A)$ contains the pair $\{\mathrm{o}, \mathrm{o}\}$, then $A$ can be identified with the bounded operator $A_{\text {oo }}$ in $H_{0}$.

If $J(A)$ contains a pair $\{r, o\}$ where $r<0$, then $A$ can be considered as an unbounded operator in $H_{0}$, with domain containing $E_{0 r} H_{r} \subset H_{0}$.

If $J(A)$ does not contain any pair $\{r, o\}(r \in I)$ then $A$ is not a reasonable operator in $H_{0}$; its domain in $H_{0}$ contains only the vector 0 . This is the case e.g. with field operators at a point (see Section 2 of the accompanying paper). Simpler examples are easy to find; so e.g. the convolution by $e^{|x|}$ is not a reasonable operator in $L^{(2)}$ (with respect to the Lebesgue measure) but it is everywhere defined in the nested Hilbert space of Example 1.1 of [1], in which $L^{(2)}=H_{0}$.

\section{Homomorphisms and related concepts}

Roughly speaking, a homomorphism is an operator $A$ with the following property: If the scalar product $\langle f \mid g\rangle$ exists, then the scalar 14 Commun. math. Phys., Vol. 4 
product $\langle A f \mid A g\rangle$ also exists. If $A$ is a homomorphism of $H_{I}$ into itself, it need not map every Hilbert space $H_{r}$ into itself. In other words, the set $J(A)$ need not contain the diagonal of $I \times I$. This is seen in the accompanying paper on the example of permutation and Poincaré operators. This discrepancy between the definition of PaLars [3] and ours is due to the fact that our $I$ is, in general, only partially ordered.

Neither homomorphisms nor arbitrary operators in $H_{I}$ coincide with the continuous mappings of the topological vector space $H_{I}^{\text {top }}$ $=\lim _{\longrightarrow}\left[H_{r} ; E_{s r} ; I\right]$, where $\lim _{\longrightarrow}$ denotes the topological inductive limit.

Many "natural embeddings" are homomorphisms. This can be seen in Section $2 b$ and $3 b$.

The definition of isomorphism and of unitary isomorphism (Section 2c) is immediate. A representation of a group $G$ in $H_{I}$ is, by definition, a homomorphic mapping of $G$ into the group $\mathscr{U}\left(H_{I} ; H_{I}\right)$ of unitary ${ }^{3}$ automorphisms of $H_{I}$.

\section{a) Homomorphism}

2.1. Definition. Let $H_{I}$ and $F_{I^{\prime}}$ be nested Hilbert spaces. An operator $A \in L\left(H_{I} ; F_{I^{\prime}}\right)$ will be called a homomorphism if it satisfies the following condition:

(Hom): For every $r \in I$ there exists at least one $r^{\prime} \in I^{\prime}$ such that $\left\{r, r^{\prime}\right\} \in$ $\in J(A)$ and $\left\{\bar{r}, \bar{r}^{\prime}\right\} \in J(A)$.

The set of all homomorphisms from $H_{I}$ into $F_{I^{\prime}}$ will be denoted by $\operatorname{Hom}\left(H_{I} ; F_{I^{\prime}}\right)$.

If $A \in L\left(H_{I} ; H_{I}\right)$ and if $J(A)$ contains the diagonal of $I \times I$, then $A \in \operatorname{Hom}\left(H_{I} ; H_{I}\right)$. (Immediate verification of (Hom)). In particular: In any nested Hilbert space, the identity operator (with representatives $\left.E_{s r}\right)$ is a homomorphism.

2.2. Proposition. Let $A \in \operatorname{Hom}\left(H_{I} ; F_{I^{\prime}}\right)$. Then the product $A^{*} A$ is defined and belongs to Hom $\left(H_{I} ; H_{I}\right)$. The set $J\left(A^{*} A\right)$ contains the diagonal of $I \times I$.

Proof. Let $\left\{r, r^{\prime}\right\} \in J(A)$ be such that $\left\{\bar{r}, \bar{r}^{\prime}\right\} \in J(A)$. Here $r$ can be arbitrarily chosen, by (Hom). Then $\left\{r^{\prime}, r\right\} \in J\left(A^{*}\right)$, by (1.5). Consequently $r^{\prime} \in R(A) \cap D\left(A^{*}\right) \neq \theta$. This means that $A^{*} A$ is defined. The representative of $A^{*} A$ between $H_{r}$ and $H_{r}$ is $\left(A^{*} A\right)_{r r}=\left(A^{*}\right)_{r r^{\prime}} A_{r^{\prime}}$. So $J\left(A^{*} A\right)$ contains the diagonal of $I \times I$ which proves that $A^{*} A$ is a homomorphism.

2.3. Proposition. For $i=1,2, \ldots$, let

$$
A^{(i)} \in \operatorname{Hom}\left(H_{I}^{(i+1)} ; H_{I}^{(i)}\right) .
$$

3 This is not the only possible definition. In the study of the complexification of representations it is natural to go back to $L\left(H_{I} ; H_{I}\right)$ where the product is not always defined. 
Then the product of $n$ factors

$$
A^{(n+1)} \ldots A^{(2)} A^{(1)}
$$

is defined and belongs to $\operatorname{Hom}\left(H_{I}^{(n+1)} ; H_{I}^{(1)}\right)$.

Proof. Notice first that (Hom) implies $D(A)=I$ for every homomorphism. So the product of $n$ homomorphisms is defined. Now start with an arbitrary $r^{(1)} \in I^{(1)}$. Choose $r^{(2)} \in I^{(2)}$ so that $\left\{r^{(1)}, r^{(2)}\right\} \in J\left(A^{(1)}\right)$ and $\left\{\bar{r}^{(1)}, \bar{r}^{(2)}\right\} \in J\left(A^{(1)}\right)$. Choose $r^{(3)}$ so that $\left\{r^{(2)}, r^{(3)}\right\} \in J\left(A^{(2)}\right)$ and $\left\{\bar{r}^{(2)}, \bar{r}^{(3)}\right\} \in J\left(A^{(2)}\right)$. Continue in this way to $\left\{r^{(n)}, r^{(n+1)}\right\} \in J\left(A^{(n)}\right)$. Then we have

and

$$
\left\{r^{(1)}, r^{(n+1)}\right\} \in J\left(A^{(n+1)} \ldots A^{(1)}\right)
$$

$$
\left\{\bar{r}^{(1)}, \bar{r}^{(n+1)}\right\} \in J\left(A^{(n+1)} \ldots A^{(1)}\right) .
$$

This proves the assertion.

2.4. Proposition. If $A^{(1)} \ldots A^{(n)}$ are as above and if $B \in L\left(H_{I}^{(0)} ; H_{I}^{(1)}\right)$, then the product of $n+1$ factors $A^{(n)} \ldots A^{(2)} A^{(1)} B$ is defined and belongs to $L\left(H_{I}^{(n+1)} ; H_{I}^{(0)}\right)$.

Proof. Immediate verification, which utilizes only the fact that $D\left(A^{(n)}\right)=I^{(n)}$ for every $n$.

2.5. The sum of two homomorphisms need not, in general, be a homomorphism.

2.6. Proposition. Let $A \in \operatorname{Hom}\left(H_{I} ; F_{I^{\prime}}\right)$. Let $h \in H_{I}, g \in H_{I}$ be such that the scalar product $\langle h \mid g\rangle$ is defined. Then the scalar product $\langle A h \mid A g\rangle$ is also defined (in $F_{I^{\prime}}$ ).

Proof. The assumption means that there exists an $r \in \bar{J}(h) \cap J(g)$; then the representatives $h_{\bar{r}}$ and $g_{r}$ exist. Let $r^{\prime}$ be such that $\left\{r, r^{\prime}\right\} \in J(A)$ and $\left\{\bar{r}, \bar{r}^{\prime}\right\} \in J(A)$. Then $A_{\bar{r}^{\prime}, \bar{r}} h_{\bar{r}}$ is a representative of $A h$ and $A_{r^{\prime} r} g_{r}$ is a representative of $A g$. Consequently $r^{\prime} \in \bar{J}(A h) \cap J(A g)$ which shows that $\langle A h \mid A g\rangle$ is defined.

2.7. Example. Let $h \in H_{I}$ be such that $J(h)=I$. Let $f \in F_{I^{\prime}}$ be such that $\mathrm{o}^{\prime} \in J(f)$. Then $|f\rangle\langle h| \in \operatorname{Hom}\left(H_{I} ; F_{I^{\prime}}\right)$. Here $|f\rangle\langle h|$ is defined as in Sec. $4 \mathrm{e}$ of [1], or Sec. 1d of this paper.

Indeed, it can be seen from Sec. 4 e of [1] that $J(|f\rangle\langle h|) \supseteqq \bar{J}(h) \times J(f)$. In our case, $J(|f\rangle\langle h|) \supseteqq I \times J(f)$. If $r$ is any element of $I$, then $\{r, o\} \in$ $\in J(|f\rangle\langle h|)$ and also $\{\bar{r}, \bar{o}\}=\{\bar{r}, o\} \in J(|f\rangle\langle h|)$. This shows that $|f\rangle\langle h| \in$ $\in \operatorname{Hom}\left(H_{I} ; F_{I^{\prime}}\right)$.

This example shows also that the adjoint of an $A \in \operatorname{Hom}\left(H_{I} ; F_{I^{\prime}}\right)$ need not belong to $\operatorname{Hom}\left(F_{I^{\prime}} ; H_{I}\right)$.

\section{b) The operator $\mathscr{E} I \tilde{I}$}

Let $H_{I}$ be any nested Hilbert space. Let $\tilde{I}$ be a subset of $I$, satisfying the following conditions: 
(i) With the order inherited from $I$, the set $\tilde{I}$ satisfies the conditions $\left(I_{1}\right),\left(I_{2}\right),\left(I_{3}\right)$ of Section $2 \mathrm{e}$ of [1].

(ii) If $\tilde{r} \in \tilde{I}$ and $\tilde{q} \in \tilde{I}$ are arbitrary, then there exists a $\tilde{p} \in \tilde{I}$ such that $\tilde{p} \leqq \tilde{r}, \tilde{q}$ and that $E_{I \tilde{p}} H_{\tilde{p}}=E_{I \tilde{r}} H_{\tilde{r}} \cap E_{I \tilde{q}} H_{\tilde{q}}$.

Consider the family $\left\{H_{\tilde{r}}\right\}(\tilde{r} \in \tilde{I})$ of Hilbert spaces and the family $\left.\left\{E_{\tilde{s} \tilde{r}}\right\} \tilde{r} \in \tilde{I}, \tilde{s} \in \tilde{I}, \tilde{s} \geqq \tilde{r}\right)$ of nestings obtained by restriction from $H_{r}$ and $E_{s r}$. It is easy to verify that the algebraic inductive limit $H_{\tilde{I}}=\left[H_{\tilde{r}} ; E_{\tilde{s} \tilde{r}} ; \tilde{I}\right]$ is a nested Hilbert space.

Denote by $\mathscr{E} I \tilde{I}$ the natural embedding of $H_{\tilde{I}}$ into $H_{I}$. It can be defined as follows:

Let $\tilde{r}$ be any element of $\tilde{I}$. Denote by $r$ the same element, considered as belonging to $I$. Then the representative $\mathscr{E}_{r \tilde{r}}$ of $\mathscr{E}$ is the identity map in $H_{r}=H_{\tilde{r}}$.

2.8. Proposition. $\mathscr{E}^{I} \tilde{I}$ is a homomorphism from $H_{\tilde{I}}$ into $H_{I}$.

Proof. Immediate verification of the condition (Hom).

Remark. Assume that $\tilde{I} \neq I$. Then there is no natural inverse homomorphism from $H_{I}$ into $H_{\tilde{I}}$, even if $\tilde{I}$ is cofinal with $I$ so that there exists a canonical bijection between vectors in $H_{I}$ and vectors in $H_{\tilde{I}}$.

\section{c) Isomorphism}

An operator $A \in \operatorname{Hom}\left(H_{I} ; F_{I^{\prime}}\right)$ is called an isomorphism if there exists a $B \in \operatorname{Hom}\left(F_{I^{\prime}} ; H_{I}\right)$ such that $B A$ is the identity in $H_{I}$ and that $A B$ is the identity in $F_{I^{\prime}}$.

If $A \in \operatorname{Hom}\left(H_{I} ; F_{I^{\prime}}\right)$ is an isomorphism, then the correspondence $h \rightarrow A h$ is bijective. Indeed, let $h \in H_{I}$ be such that $A h=0$. Then $B A h=h=0$ which shows that $A$ is injective. Furthermore, every $f \in F_{I}$ can be written as $f=A B f$, which completes the proof.

We are here using the fact that the product of three factors $A B f$ is defined. (See Section 1c, and Proposition 2.3.)

An operator $U \in L\left(H_{I} ; F_{I^{\prime}}\right)$ is said to be unitary if $U^{*} U$ and $U U^{*}$ are defined, and if $U^{*} U=1$ (identity in $H_{I}$ ) as well as $U U^{*}=1$ (identity in $F_{I^{\prime}}$ ).

A unitary operator need not be an isomorphism. For example, let $U_{0^{\prime} 0}$ be an arbitrary unitary mapping from the Hilbert space $H_{0}$ onto the Hilbert space $F_{0^{\prime}}$. Then the operator $E_{2}\left(I \times I^{\prime} ;\right.$ o, o $\left.o^{\prime}\right) U_{0^{\prime}}$ o (i.e. the element of $L\left(H_{I} ; F_{I^{\prime}}\right)$ whose $\left\{0, o^{\prime}\right\}$-representative is $\left.U_{0^{\prime}}\right)$ is unitary but - in general - not an isomorphism.

An isomorphism which is also unitary will be called unitary isomorphism. The set of unitary isomorphisms from $H_{I}$ onto $F_{I^{\prime}}$ will be denoted by $\mathscr{U}\left(H_{I} ; F_{I^{\prime}}\right)$. 
Let $h \in H_{I}$ and $g \in H_{I}$ be such that $\langle h \mid g\rangle$ is defined. Let $U \in \mathscr{U}\left(H_{I} ; F_{I^{\prime}}\right)$. Then $\langle U h \mid U g\rangle$ is also defined, and

$$
\langle U h \mid U g\rangle=\langle h \mid g\rangle
$$

as can be immediately verified.

If $U$ is only assumed to be unitary then the existence of $\langle h \mid g\rangle$ need not entail the existence of $\langle U h \mid U g\rangle$; if $\langle U h \mid U g\rangle$ exists, then it is equal to $\langle h \mid g\rangle$.

\section{Direct sums}

The infinite direct sums to be defined now are not algebraic. A vector can belong to $\bigoplus_{\mathrm{n}=0}^{\infty} H_{I}^{(n)}$ even if it has infinitely many non-zero components.

The conditions under which a direct sum is defined (Section 3 a) are not restrictive. They could have been incorporated into the definition of nested Hilbert space. The definition utilizes all (Hilbert) direct sums that can be built from the Hilbert summands. Here again, the considerations of only totally ordered $I$-s would have been too restrictive.

Section $3 \mathrm{~b}$ is devoted to the operator of natural embedding of a summand into the sum. Its adjoint is the projection of the sum onto the summand. These operators are homomorphisms in the sense of Section $2 \mathrm{a}$.

These results are used in the accompanying paper in the construction of Fock spaces.

\section{a) Direct sum of nested Hilbert spaces}

For $n=0,1,2, \ldots$ let $I^{(n)}$ be an ordered set that satisfies the conditions $\left(I_{1}\right),\left(I_{2}\right),\left(I_{3}\right)$ of Sec. $2 \mathrm{e}$ of $[1]$. Define $\underline{I}=\left\{I^{(0)}, I^{(1)}, \ldots I^{(n)}, \ldots\right\}$ as the set of sequences

$$
\underline{r}=\left\{r^{(0)}, \ldots r^{(n)}, \ldots\right\} \quad\left(r^{(n)} \in I^{(n)}, \quad n=0,1,2, \ldots\right) .
$$

Define in $\underline{I}$ an order by writing $\underline{s} \geqq \underline{r}$ if and only if $s^{(n)} \geqq r^{(n)}$ for every $n$.

It is easy to verify that $\underline{I}$ satisfies the conditions $\left(I_{1}\right),\left(I_{2}\right),\left(I_{3}\right)$ of Sec. $2 \mathrm{e}$ of $[1]$, with $\bar{r}=\left\{\bar{r}^{(0)}, \ldots \bar{r}^{(n)}, \ldots\right\}$ and $\underline{Q}=\left\{\mathrm{o}^{(0)}, \ldots, \mathrm{o}^{(n)}, \ldots\right\}$.

For $n=0,1,2, \ldots$, let $H_{I}^{(n)}=\left[H_{r}^{(n)} ; E_{s r}^{(n)} ; I^{(n)}\right]$ be nested Hilbert spaces. (For the sake of typographical simplicity, the superscript $n$ appears only once in - say - $E_{s r}^{(n)}$; strictly speaking the notation should be $E^{(n)}\left(s^{(n)} ; r^{(n)}\right)$ with $r^{(n)} \in I^{(n)}$ and $s^{(n)} \geqq r^{(n)}$.)

Assume:

$\left(D S_{1}\right)$. For any pair $\underline{r} \in \underline{I}, \underline{s} \in \underline{I}$ such that $\underline{s} \geqq \underline{r}$ there exists a constant $\gamma=\gamma(\underline{s} ; \underline{r})$ (independent of $n$ ) such that

$$
\left\|E_{s r}^{(n)}\right\| \leqq \gamma \quad(n=0,1,2, \ldots) .
$$

Let $\underline{r} \in \underline{I}$ and $q \in \underline{I}$ be arbitrary. For every $n$, let $p^{(n)} \leqq r^{(n)}, q^{(n)}$ be such that $E_{I r}^{(n)} H_{r}^{(n)} \cap E_{I q}^{(n)} H_{q}^{(n)}=E_{I p}^{(n)} H_{p}^{(n)}$. Consider the bijective bicontinuous operator $E_{[r q] p}^{(n)}$ studied in Sec. $3 \mathrm{~b}$ of [1]. Assume 
$\left(D S_{2}\right)$. If $\underline{r}, \underline{q}$ are arbitrary, then there exists a $\underline{p} \leqq \underline{r}, \underline{q}$ such that, for some constant $k=k(r, q$ ) (independent of $n$ ) we have

$$
\left\|\left(E_{[r q] p}^{(n)}\right)_{p[r q]}^{-1}\right\|<k .
$$

For every $\underline{r} \in \underline{I}$, define a Hilbert space $H_{\underline{\underline{r}}}$ as the Hilbert direct sum

That is :

$$
H_{\underline{r}}=\bigoplus_{n=0}^{\infty} H_{r}^{(n)} \quad\left(\underline{r}=\left\{r^{(0)}, \ldots, r^{(n)}, \ldots\right\}\right) .
$$

An element of $H_{\underline{r}}$ is a sequence

such that

$$
f_{\underline{r}}=\left\{f_{r}^{(0)}, \ldots, f_{r}^{(n)}, \ldots\right\}
$$

$$
\sum_{n=0}^{\infty}\left(f_{r}^{(n)}, f_{r}^{(n)}\right)<\infty
$$

(Here again, the correct notation would be $f^{(n)}\left(r^{(n)}\right)$ ).

For $\underline{s} \geqq \underline{r}(\underline{r} \in \underline{I})$ define $E_{\underline{s} \underline{r}}$ as the direct sum

That is:

$$
E_{\underline{s} \underline{r}}=\bigoplus_{n=0} E_{s r}^{(n)}
$$

for every $f_{\underline{r}} \in H_{\underline{r}}$.

$$
E_{\underline{s} \underline{r}} f_{\underline{r}}=\left\{E_{s r}^{(0)} f_{r}^{(0)}, \ldots E_{s r}^{(n)} f_{r}^{(n)}, \ldots\right\}=\bigoplus_{n=0} E_{s r}^{(n)} f_{r}^{(n)}
$$

3.1. Proposition. $E_{\underline{s} \underline{r}}$ is a nesting from $H_{\underline{r}}$ into $H_{\underline{s}}$.

Proof. (a) $E_{\underline{s} \underline{r}}$ is bounded. Indeed,

$$
\left(E_{\underline{s} \underline{r}} f_{\underline{r}}, E_{\underline{s} \underline{r}} f_{\underline{r}}\right)=\sum_{n=0}^{\infty}\left(E_{s r}^{(n)} f_{r}, E_{s r}^{(n)} f_{r}\right) \leqq \gamma^{2} \sum_{n=0}^{\infty}\left(f_{r}^{(n)}, f_{r}^{(n)}\right)=\gamma^{2}\left\|f_{\underline{r}}\right\|^{2} .
$$

(b) $E_{\underline{s} \underline{r}}$ is injective: $E_{\underline{s} \underline{r}} f_{\underline{r}}=0$ means $E_{s r}^{(n)} f_{r}^{(n)}=0$ for every $n$. Since all the $\bar{E}_{s r}^{(n)}$ are injective it follows that $f_{r}^{(n)}=0(n=0,1,2, \ldots)$ i.e. that $f_{r}=0$.

(c) The range of $E_{\underline{s} \underline{r}}$ is dense in $H_{\underline{s}}$. Let $g_{\underline{s}} \in H_{\underline{s}}$ be such that $\left(g_{\underline{s}}, E_{\underline{s}} f_{\underline{r}}\right)$ $=\sum_{n=0}^{\infty}\left(g_{s}^{(n)}, E_{s r}^{(n)} f_{r}^{(n)}\right)=0$ for every $f_{\underline{r}} \in H_{\underline{r}}$. Then in particular $\left(g_{\underline{s}}, E_{\underline{s} \underline{r}} f_{\underline{r}}\right)=0$ for every $f_{\underline{r}}$ of the form $f_{\underline{r}}=\left\{0,0, \ldots, 0, f_{r}^{(n)}, 0, \ldots\right\}\left(f_{r}^{(n)} \neq 0\right)$. It follows that $g_{s}^{(n)}=0,(n=0,1,2, \ldots)$ i.e. that $g_{\underline{s}}=0$. This proves the proposition.

It can immediately be verified that the family $E_{\underline{r} \underline{s}}(\underline{r}, \underline{s} \in I, \underline{s} \geqq \underline{r})$ satisfies the conditions $\left(\operatorname{Ind}_{1}\right),\left(\operatorname{Ind}_{2}\right)$ of Sec. $2 \mathrm{f}$ of [1].

Denote by $H_{I}$ the algebraic inductive limit of the Hilbert spaces $H_{\underline{r}}$ with respect to the nestings $E_{\underline{s}}$.

3.2. Proposition. There exists a canonical bijective correspondence between elements of $H_{\underline{I}}$ and sequences

$$
\left\{f^{(0)} \ldots f^{(n)} \ldots\right\} \quad\left(f^{(n)} \in H_{I}^{(n)}\right)
$$


which have the following property: For at least one $\underline{\underline{ }} \in \underline{I}$, the sum $\sum_{n=0}^{\infty}\left(f_{r}^{(n)}, f_{r}^{(n)}\right)$ is finite. If $\underline{v} \in J(f)$ and if $f_{\underline{r}}=\sum_{n} f_{r}^{(n)} \in \bigoplus_{n=0}^{\infty} H_{r}^{(n)}$ is the representative of $f$ in $H_{\underline{r}}$, then the sequence corresponding to $f$ is $\left\{E_{I r}^{(0)} f_{r}^{(0)}, \ldots, E_{I r}^{(n)} f_{r}^{(n)}, \ldots\right\}$. We shall write $f=\left\{E_{I r}^{(0)} f_{r}^{(0)}, \ldots E_{I r}^{(n)} f_{r}^{(n)}, \ldots\right\}$.

Proof. (a) Let $f \in H_{\underline{I}}$ and $\underline{r} \in J(f)$. Then the representative $f_{\underline{r}}$ of $f$ in $H_{\underline{r}}$ is an element of the Hilbert direct sum $H_{\underline{r}}=\bigoplus_{n=0}^{\infty} H_{r}^{(n)}$, i.e. a sequence $\left\{f^{(n)}\right\}(n=0,1,2 \ldots)$ such that

$$
\sum_{n=0}\left(f_{r}^{(n)}, f_{r}^{(n)}\right)<\infty .
$$

Consider the sequence $\left\{f^{(n)}\right\}$, where $f^{(n)}=E_{I r}^{(n)} f_{r}^{(n)}$. It obviously has the property described in the proposition, and is independent of the choice of $\underline{r} \in J(f)$.

(b) Let $\left\{f^{(0)}, \ldots f^{(n)} \ldots\right\}$ be a sequence satisfying the condition of the proposition. If $\underline{r} \in \underline{I}$ is such that (3.3) holds, then the sequence $f_{r}^{(n)}$ belongs to $\bigoplus_{n} H_{r}^{(n)}=H_{\underline{r}}$. Consequently $E_{I \underline{r}}\left\{f_{r}^{(n)}\right\}$ belongs to $H_{\underline{I}}$. We can write $E_{\underline{I} \underline{r}}\left\{f_{\underline{x}}^{(n)}\right\}=\left\{E_{l r}^{(n)} f_{r}^{(n)}\right\}=\left\{f^{(n)}\right\}$.

This proves the proposition.

3.3 Theorem. $H_{\underline{I}}$ is a nested Hilbert space.

Proof. (a) For every $\underline{\underline{r}} \in \underline{I}$, define $u_{\underline{\underline{\underline{r}}} \underline{\underline{r}}}$ as $\bigoplus_{n} u_{\vec{r} r}^{(\underline{r})}$. The condition $\left(\mathrm{NH}_{2}\right)$ of Sec. $3 \mathrm{a}$ of $[1]$ can easily be verified.

(b) In order to verify $\left(N H_{1}\right)$, let $\underline{r} \in \underline{I}$ and $q \in I$ be arbitrary. For every $n$, let $p^{(n)} \leqq r^{(n)}, q^{(n)}$ be such that $E_{I r}^{(n)} H_{r}^{(n)} \cap E_{I q}^{(n)} H_{q}^{(n)}=E_{I p}^{(n)} H_{p}^{(n)}$ and that the condition $\left(D S_{2}\right)$ holds. We shall show that $E_{\underline{I}} \underline{\underline{p}} H_{\underline{p}}=E_{\underline{I} \underline{\underline{r}}} H_{\underline{\underline{x}}} \cap$ $\cap E_{\underline{I} \underline{q}} H_{\underline{q}}$. It is clear that $E_{\underline{I} \underline{p}} H_{\underline{p}}^{\subseteq} E_{\underline{I} \underline{r}} H_{\underline{r}} \cap E_{\underline{I} \underline{q}} H_{\underline{q}}$ since $\underline{p} \leqq \underline{r}, \underline{q}$. In order to prove the equality, consider an arbitrary $t \in E_{\underline{I} \underline{\underline{r}}} \bar{H}_{\underline{\underline{x}}} \cap E_{\underline{I} \underline{q}} H_{\underline{q}}$. Then $t_{r}=\left\{t_{r}^{(n)}\right\}$ and $t_{q}=\left\{t_{q}^{(n)}\right\}$ where $\sum_{n=0}^{\infty}\left(t_{r}^{(n)}, t_{r}^{(n)}\right)<\infty$ and

$$
\sum_{n=0}^{\infty}\left(t_{q}^{(n)}, t_{q}^{(n)}\right)<\infty \text {. }
$$

By the definition of $p$, every $t^{(n)} \in E_{I r}^{(n)} t_{r}^{(n)} \cap E_{I q}^{(n)} t_{q}^{(n)}$ has a representative in $H_{p}^{(n)}$. So $t_{r}^{(n)}=E_{r p}^{(n)} t_{p}^{(n)}$ and $t_{q}^{(n)}=E_{q p}^{(n)} t_{p}^{(n)}$, which means

$$
\left(\begin{array}{l}
t_{r}^{(n)} \\
t_{q}^{(n)}
\end{array}\right)=E_{[r q] p}^{(n)} t_{p}^{(n)}
$$

By $\left(D S_{2}\right)$, there exists a constant $k$ (independent of $n$ ) such that

$$
\left\|t_{p}^{(n)}\right\|^{2} \leqq k^{2}\left(\left\|t_{r}^{(n)}\right\|^{2}+\left\|t_{q}^{(n)}\right\|^{2}\right)
$$

It follows that $\left\{t_{p}^{(n)}\right\} \in \bigoplus_{n=0} H_{p}^{(n)}=H_{\underline{p}}$. So $t$ has a representative $t_{\underline{p}}$ in $H_{\underline{p}}$ which means $t \in E_{\underline{I} \underline{p}} H_{\underline{p}}$ and proves the theorem. 


\section{b) The operators $\mathscr{I}^{(n)}$ and $\mathscr{P}(n)$}

Let $H_{I}^{(n)}(n=0,1,2, \ldots)$ be nested Hilbert spaces. Assume that the conditions $\left(D S_{1}\right)$ and $\left(D S_{2}\right)$ are satisfied, so that the direct sum $H_{\underline{I}}=\bigoplus_{n=0}^{\infty} H_{I}^{(n)}$ is defined.

We shall now study the natural embedding of $H_{I}^{(n)}$ into $H_{I}$ considered as an element of $L\left(H_{I}^{(n)} ; H_{I}\right)$. This operator will be denoted by $\mathscr{I}(n)$.

3.4. Proposition. Let $r^{(n)} \in I^{(n)}$ be arbitrary and let

$$
\underline{q}=\left\{q^{(0)}, \ldots q^{(n)}, \ldots\right\} \in \underline{I}
$$

be such that $q^{(n)} \geqq r^{(n)}$. Consider the bounded operator $\mathscr{I}_{\underline{q} r}^{(n)}$ from $H_{r}^{(n)}$ into

$$
\begin{aligned}
H_{\underline{q}}=\bigoplus_{n=0}^{\infty} H_{q}^{(n)}, \text { defined by } & \\
\mathscr{I}_{\underline{q} r}^{(n)} f_{r}^{(n)} & =\left\{0,0, \ldots 0, E_{q r}^{(n)} f_{r}^{(n)}, 0, \ldots\right\} \\
& =\left\{0,0, \ldots 0, f_{q}^{(n)}, 0, \ldots\right\} .
\end{aligned}
$$

Then $\mathscr{I}_{q \underline{r}}^{(n)}$ is the $r_{\underline{\underline{q}}}^{(n)}$-representative of an operator $\mathscr{I}(n) \in L\left(H_{I}^{(n)} ; H_{\underline{I}}\right)$ which is independent of the choice of $r^{(n)}$ and of $q$ (subject to the condition $\left.q^{(n)} \geqq r^{(n)}\right)$. It satisfies

for every $f^{(n)} \in H_{I}^{(n)}$.

$$
\begin{aligned}
D(\mathscr{I}(n)) & =I^{(n)} \\
R\left(\mathscr{I}^{(n)}\right) & =\underline{I} \\
\mathscr{I}^{(n)} f^{(n)} & =\left\{0, \ldots 0, f^{(n)}, 0, \ldots\right\}
\end{aligned}
$$

Proof. (a) Let $r^{(n)} \in I^{(n)}$ and $q \in \underline{I}$ be such that $q^{(n)} \geqq r^{(n)}$. Let $\underline{s} \geqq \underline{q}$ and $p^{(n)} \leqq r^{(n)}$. Then, for every $\tilde{f}_{p}^{(n)} \in H_{p}^{(n)}$,

$$
\begin{aligned}
& E_{\underline{\underline{g}} \underline{q}} \mathscr{J}_{\underline{q} r}^{(n)} E_{r p}^{(n)} f_{p}^{(n)}=E_{\underline{\underline{s}} \underline{q}} \mathscr{I}_{\underline{\underline{q}} \underline{r}}^{(n)} f_{r}^{(n)} \\
& =E_{\underline{s} \underline{q}}\left\{0, \ldots 0, f_{q}^{(n)}, 0, \ldots\right\}=\left\{0, \ldots 0, E_{s q}^{(n)} f_{q}^{(n)}, 0, \ldots\right\} \\
& =\left\{0, \ldots 0, f_{s}^{(n)}, 0, \ldots\right\}=\mathscr{I}_{\underline{s} p}^{(n)} f_{p}^{(n)} .
\end{aligned}
$$

(b) Let $r^{(n)}, \hat{r}^{(n)} \in I^{(n)}$ and $\underline{q}, \underline{\hat{q}} \in \underline{I}$ be such that $r^{(n)} \geqq q^{(n)}$ and $\hat{r}^{(n)} \geqq \hat{q}^{(n)}$. Let $p^{(n)}$ be a common predecessor of $r^{(n)}$ and of $\hat{r}^{(n)}$; let $\underline{s}$ be a common successor of $\underline{q}$ and of $\underline{\hat{q}}$. Then, by (a),

$$
E_{\underline{s} \underline{q}} \mathscr{J}_{\underline{q} r}^{(n)} E_{r p}^{(n)}=\mathscr{I}_{\underline{s} p}^{(n)}=E_{\underline{s} \underline{\underline{q}}} \mathscr{I}_{\underline{\underline{q}} \hat{r}}^{(n)} E_{\hat{r} p}^{(n)}
$$

which proves that $\mathscr{I}_{\underline{\underline{q}}}^{(n)}$ and $\mathscr{I}_{\underline{\underline{q}} \hat{r}}^{(n)}$ are representatives of one and the same $\mathscr{I}(n) \in L\left(H_{I}^{(n)} ; H_{\underline{I}}\right)$.

(c) The set $J\left(\mathscr{I}^{(n)}\right)$ contains, by (a) and (b), all the pairs $\left\{r^{(n)}, \underline{r}\right\}$ where $r^{(n)} \in I^{(n)}$ is arbitrary and where $\underline{r} \in \underline{I}$ is such that $r^{(n)}$ is the $n$-th component of $\underline{r}$. This shows, in particular, that (3.5) and (3.6) hold.

(d) Let $f^{(n)} \in H_{I}^{(n)}$ be arbitrary. Let $r^{(n)} \in J\left(f^{(n)}\right)$ and $q \in I$ be such that $q^{(n)} \geqq r^{(n)}$ so that $\left\{r^{(n)}, \underline{q}\right\} \in J(\mathscr{I}(n))$. Then (3.4) follows from (a) and (b).

This proves the proposition. 
Define $\mathscr{P}^{(n)} \in L\left(H_{\underline{I}} ; H^{(n)}\right)$ as the adjoint of $\mathscr{I}^{(n)}$. That is:

$$
\mathscr{P}(n)=(\mathscr{I}(n))^{*}
$$

which means, by (1.5), that

$$
\mathscr{P}_{\bar{r}} \overline{\underline{q}} \bar{q}=u_{\bar{r} r}^{(n)}\left(\mathscr{I}_{\underline{q} r}^{(n)}\right)_{r \underline{q}}^{*} u_{\underline{q} \bar{q}}
$$

for every $\bar{q}$ and for every $\bar{r}^{(n)}$ such that $\bar{r}^{(n)} \geqq \bar{q}^{(n)}$. It follows from (3.5) and (3.6), that

$$
\begin{aligned}
& D\left(\mathscr{P}^{(n)}\right)=\underline{I} \\
& R\left(\mathscr{P}^{(n)}\right)=I^{(n)} .
\end{aligned}
$$

3.5. Proposition. If $g=\left\{g^{(0)}, \ldots g^{(n)}, \ldots\right\}$ is any element of $H_{\underline{I}}$ then $\mathscr{P}^{(n)} g$ is defined and

$$
\mathscr{P}(n) g=g^{(n)} .
$$

Proof. (a) It follows from (3.11) that $\mathscr{P}(n) \mathrm{g}$ is defined.

(b) If $\underline{q} \in J(g)$ and if $\bar{r}^{(n)} \geqq \bar{q}^{(n)}$ then

$$
P \frac{(n)}{r} \underline{\underline{q}} g_{\underline{q}}=g_{\frac{(n)}{r}} \text {. }
$$

Indeed, let $f_{\frac{(n)}{r}}^{(n)}$ an arbitrary element of $H_{\frac{(n)}{r}}^{(\text {Then }}$

$$
\begin{aligned}
& \left(f_{\bar{r}}^{(n)}, P_{r \underline{q}}^{(n)} g_{\bar{q}}\right)=\left(f_{\bar{r}}^{(n)}, u_{\bar{r} r}^{(n)}\left(\mathscr{I}_{\underline{q} r}^{(n)}\right)_{r \underline{q}}^{*} u_{q \underline{q}} g_{\underline{q}}\right) \\
& =\left(u_{\bar{q} q} \mathscr{I}_{\underline{q} r}^{(n)} u_{r \bar{r}}^{(n)} f_{\bar{r}}^{(n)}, g_{\bar{q}}\right)=\left(u_{\bar{q} q}^{(n)} E_{q r}^{(n)} u_{r \bar{r}}^{(n)} f_{\bar{r}}^{(n)}, g_{\bar{q}}^{(n)}\right) \\
& =\left(f_{\bar{r}}^{(n)}, u_{r r}^{(n)}\left(E_{q r}^{(n)}\right)_{r q}^{*} u_{q \bar{q}}^{(n)} g_{\bar{q}}^{(n)}\right)=\left(f_{\bar{r}}^{(n)}, E_{\bar{r}}^{(n)} g_{\bar{q}}^{(n)}\right)=\left(f_{\bar{r}}^{(n)}, g_{\bar{r}}^{(n)}\right)
\end{aligned}
$$

which completes the proof of the proposition.

3.6. Proposition. For every $n$ and every $m(n, m$, integers $\geqq 0)$, one has

$$
\begin{aligned}
& \mathscr{I}(m) \mathscr{P}(n)=\delta_{m n} P^{(n)} \in \operatorname{Hom}\left(H_{\underline{I}} ; H_{\underline{I}}\right) \\
& \mathscr{P}(n) \mathscr{I}(m)=\delta_{m n} 1^{(n)} \in \operatorname{Hom}\left(H_{I}^{(n)} ; H_{I}^{(n)}\right) .
\end{aligned}
$$

Here $\delta_{m n}$ is the Kronecker symbol. The operator $P^{(n)} \in \operatorname{Hom}\left(H_{\underline{I}} ; H_{\underline{I}}\right)$ satisfies

and

$$
\left(P^{(n)}\right)^{*}=P^{(n)}
$$

$$
\left(P^{(n)}\right)^{2}=P^{(n)} .
$$

In $(3.13), 1^{(n)}$ stands for the identity operator (with representatives $\left.E_{s r}^{(n)}\right)$ in $H_{I}^{(n)}$.

Proof. (a) If $m \neq n$, then $\mathscr{I}^{(m)} \mathscr{P}(n)=0$. This follows from (3.4) and from (3.11). Similarly $\mathscr{P}^{(n)} \mathscr{I}(m)=0$.

(b) For every $n$, one has $\mathscr{P}^{(n)} \mathscr{I}^{(n)} f^{(n)}=\mathscr{P}^{(n)}\left\{0, \ldots, 0, f^{(n)}, 0, \ldots\right\}$ $=f^{(n)}$ which proves $(3.13)$.

(c) (3.14) follows from (3.7).

(d) $\left(P^{(n)}\right)^{2}=\mathscr{I}^{(n)} \mathscr{P}(n) \mathscr{I}^{(n)} \mathscr{P}(n)=\mathscr{I}^{(n)} \mathscr{P}(n)=P^{(n)}$ which proves $(3.15)$.

Notice finally that $\mathscr{I}(n)$ belongs to $\operatorname{Hom}\left(H_{I}^{(n)} ; H_{\underline{I}}\right)$ and that $\mathscr{P}(n)$ belongs to $\operatorname{Hom}\left(H_{I} ; H_{I}^{(n)}\right)$. Indeed, $J\left(\mathscr{I}^{(n)}\right)$ contains all pairs $\left\{r^{(n)}, \underline{r}\right\}$, 
where $r^{(n)}$ is arbitrary and $\underline{r}$ is such that $r^{(n)}$ is the $n$-th component of $\underline{r}$. Then $\bar{r}^{(n)}$ is the $n$-th component of $\underline{\underline{r}}$, which verifies (Hom).

This concludes the proof of the proposition.

Acknowledgments. I would like to thank M. L. МотсHane for his kind hospitality at the IHES.

\section{Literature}

[1] Grossmann, A.: Commun. Math. Phys. 2, 1 (1966).

[2] Mrtchell, B.: Theory of categories, Chapter I. New York and London: Academic Press 1965.

[3] Palais, R.: Seminar on the Atiyah-Singer Index Theorem: Chapter VIII Annals of Mathematics Studies 57. Princeton 1965.

[4] Roberts, J. E.: Commun. Math. Phys. 3, 98-119 (1966). 